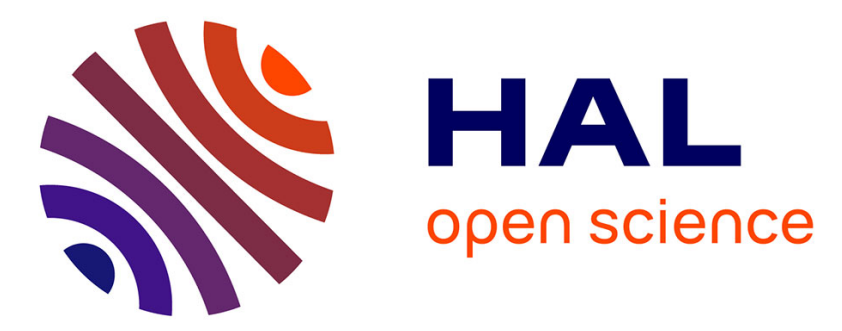

\title{
Modeling of heat flow and effective thermal conductivity of fractured media: Analytical and numerical methods
}

\author{
Sy Tuan Nguyen, Manh Huyen Vu, Minh Ngoc Vu, Anh Minh A.M. Tang
}

\section{To cite this version:}

Sy Tuan Nguyen, Manh Huyen Vu, Minh Ngoc Vu, Anh Minh A.M. Tang. Modeling of heat flow and effective thermal conductivity of fractured media: Analytical and numerical methods. Journal of Applied Geophysics, 2017, 140, pp.117-122. 10.1016/j.jappgeo.2017.03.018 . hal-01515979

HAL Id: hal-01515979

https://hal-enpc.archives-ouvertes.fr/hal-01515979

Submitted on 16 May 2019

HAL is a multi-disciplinary open access archive for the deposit and dissemination of scientific research documents, whether they are published or not. The documents may come from teaching and research institutions in France or abroad, or from public or private research centers.
L'archive ouverte pluridisciplinaire HAL, est destinée au dépôt et à la diffusion de documents scientifiques de niveau recherche, publiés ou non, émanant des établissements d'enseignement et de recherche français ou étrangers, des laboratoires publics ou privés. 
1 Modeling of heat flow and effective thermal conductivity of fractured media: analytical and numerical methods

S.T. Nguyen ${ }^{\left(a, b,{ }^{*}\right)}, M .-H . V u^{(b)}, M . N . V u^{(b)}$ and A.M. Tang ${ }^{(c)}$

(a) Euro-Engineering, Pau, France

(b) R\&D Center, Duy Tan University, Da Nang, Viet Nam

(c) Université Paris-Est, Laboratoire Navier (ENPC-IFSTTAR-CNRS) 77455 Marne la Vallée, France

(*) Corresponding author: stuan.nguyen@gmail.com

\section{Abstract}

The present work aims at modeling the thermal conductivity of fractured materials using homogenization-based analytical and pattern-based numerical methods. These materials are considered as a network of cracks distributed inside a solid matrix. Heat flow through such media is perturbed by the crack system. The problem of heat flow across a single crack is firstly investigated. The classical Eshelby's solution, extended for the calculation of the conductivity of a mixture of an ellipsoidal inclusion in an infinite homogeneous matrix, gives an analytical solution of temperature discontinuity across a non-conducting penny-shape crack. This solution is then validated by the numerical simulation based on the finite elements method. The numerical simulation allows analyzing the effect of crack conductivity. The problem of a single crack is then extended to media containing multiple cracks. Analytical estimations for effective thermal conductivity, that take into account the interaction between cracks and their spatial distribution, are developed for the case of non-conducting cracks. Pattern-based numerical method is then employed for both cases non-conducting and conducting cracks. In the case of non-conducting cracks, numerical and analytical methods, both account for the spatial distribution of the cracks, fit perfectly. In the case of conducting cracks, the numerical analyzing of crack conductivity effect shows that highly conducting cracks weakly affect heat flow, and the effective thermal conductivity of fractured media. 
Keywords: morphologically representative pattern; thermal conductivity; homogenization; fractured rock

\section{Introduction}

Thermal conductivity is an important geophysical property of rocks and largely investigated in geo-sciences such as, nuclear waste disposal, geothermal production, $\mathrm{CO}_{2}$ storage, hydrocarbon formation processes, etc (Tang and Cui, 2009; Tang et al., 2008; Cui et al, 2011). This parameter is generally affected by natural cracks distributed in the geomaterials. The homogenization-based analytical approach has been confirmed to be a powerful tool to estimate effective properties of heterogeneous materials (Eshelby, 1957; Mori and Tanaka, 1973; Giraud et al., 2007; Zimmerman, 1989). The macroscopic mechanical properties is affected by the properties of each phase in the mixture, the shape and the orientation of the particles as well as the stress acting on the considered materials. Nguyen and colleagues successfully employed this technique for the simulation of effective viscoelastic properties of fractured media (Nguyen et al., 2011, Nguyen, 2014a; Nguyen et al., 2015c) and effective elastic properties and electrical conductivity of sandstone (Nguyen, 2014b, Nguyen et al, 2015a,b).

Besides, the numerical approach based on the pattern-based method (PBM) is also used to simulate overall properties of heterogeneous materials (Bornert, 1996; Stolz and Zaoui, 1991). This approach is more powerful than the classical numerical finite element method (FEM) that simulates the whole representative elementary volume (REV) of the medium, in term of calculation time. Actually, the PBM considers a morphologically representative pattern (MRP) of the medium instead of the REV. For the case of fractured media, MRP contains only one crack whereas REV contains a whole system of micro-cracks (Pouya et al., 2013; Camacho and Ortiz, 1996). However, by managing the boundary condition and the shape of the MRP, the pattern-based method allows accounting for the interaction between cracks and their spatial distribution (Nguyen and Dormieux, 2014). 
This paper focuses on the thermal conductivity of fractured materials based on homogenization-based analytical method and PBM. The problem of heat flow through a medium containing a single crack is firstly considered. The classical Eshelby's theory, extended for the conductivity of a mixture of an ellipsoidal inclusion in an infinite homogeneous matrix, gives an analytical solution of temperature discontinuity across a nonconducting penny-shape crack. This analytical solution is then compared with the numerical simulation based on PBM. The effect of crack conductivity is also analyzed with the help of the numerical simulation. Secondly, the problem of single crack is extended to a medium containing multiple cracks. Analytical estimations of effective thermal conductivity for the case of non-conducting cracks, that accounts the interaction between the cracks and their spatial distribution, are developed. PBM is then employed for both cases, non-conducting and conducting cracks.

\section{Notations}

- $\boldsymbol{A}$ is the second order temperature field localization tensor

- $\mathbf{1}$ is the second order unit tensor

- $[T]$ is the temperature jump across a crack

- $[t]$ is the dimensionless temperature jump across a crack

- $\quad \underline{\nabla T}$ is the temperature gradient

- $\underline{z}$ is the position vector of a point

- $f$ is the volume fraction

- $\quad C$ is the conductivity

- $Q$ is the anisotropic parameters of the inclusion

- $X$ and $X_{d}$ are the aspect ratio of the cracks and of the spatial distribution of the cracks respectively

The exponents and index

- $s$ is for the solid phase

- $c$ is for crack 
- $T$ is for transversal component of the transversely isotropic tensors

- $\quad N$ is for normal component of the transversely isotropic tensors

- $m t$ is for Mori-Tanaka scheme

- $\quad c w$ is for Castañeda-Willis scheme

\section{Heat flow across a single crack}

One considers a basic problem of a single crack in a homogenous medium under a far-field homogenous temperature gradient condition: $\forall \underline{z} \rightarrow \infty: T=\underline{\nabla T} \cdot \underline{\mathrm{z}}$ (see Fig. 1). Heat flow is locally perturbed around the crack due to the contrast between the conductivity of the crack and that of the surrounding solid matrix. Temperature is discontinued across the crack.

$$
\forall \underline{\mathrm{z}} \rightarrow \infty: T=\underline{\nabla T} \cdot \underline{\mathrm{z}}
$$

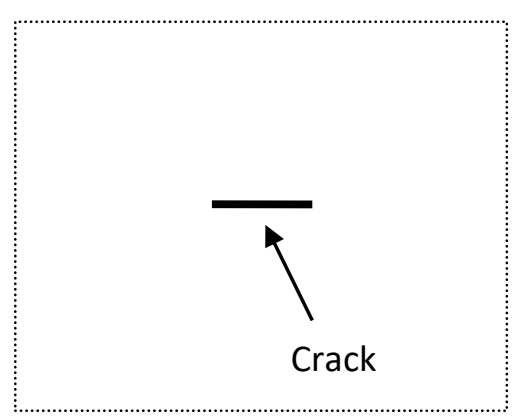

Figure1: Single crack in homogenous medium under far-field homogeneous temperature gradient boundary condition.

Note that for penny-shape crack, an extension of Eshelby's theory (Eshelby, 1957) for the problem of heat flow yields a temperature field localization tensor $\boldsymbol{A}$ that is determined by (Giraud, 2007; Nguyen, 2014):

$$
\boldsymbol{A}=\frac{C_{s}}{(1-Q) C_{s}+Q C_{c}}\left(\mathbf{1}-\underline{e}_{3} \otimes \underline{e}_{3}\right)+\frac{C_{s}}{2 Q C_{s}+(1-2 Q) C_{c}} \underline{e}_{3} \otimes \underline{e}_{3}
$$

where $C_{s}$ (resp. $C_{c}$ ) is the conductivity of the solid matrix (resp. the conductivity of the crack), $\underline{e}_{3}$ the unit normal to the crack plan, and $Q$ the geometry factor defined by: 


$$
Q=\frac{1}{2}-\frac{\sqrt{1-X^{2}}-X \arctan \left(\frac{\sqrt{1-X^{2}}}{X}\right)}{2\left(1-X^{2}\right)^{3 / 2}}
$$

98

99

Introducing eq. (3) into eq. (1) yields:

$$
\boldsymbol{A}=\frac{C_{s}}{\left(1-\frac{\pi}{4} X\right) C_{s}+\frac{\pi}{4} X C_{c}}\left(\mathbf{1}-\underline{e}_{3} \otimes \underline{e}_{3}\right)+\frac{C_{s}}{\frac{\pi}{2} X C_{s}+\left(1-\frac{\pi}{2} X\right) C_{c}} \underline{e}_{3} \otimes \underline{e}_{3}
$$

101 The local temperature gradient inside the crack, $\underline{\nabla T_{C}}$ is homogeneous and is linearly related 102 to the far-field temperature gradient (Fig. 1) as:

$$
\underline{\nabla T_{c}}=\boldsymbol{A} \cdot \underline{\nabla T}
$$

103 Its component normal to the crack plan is expressed as:

$$
\nabla T_{c, 3}=\frac{C_{s}}{\frac{\pi}{2} X C_{s}+\left(1-\frac{\pi}{2} X\right) C_{c}} \nabla T_{3}
$$

104 According to this solution, the temperature jump across the crack $[T]$ is calculated as:

$$
[T]=\nabla T_{c, 3} d=\left(\frac{C_{s}}{\frac{\pi}{2} X C_{s}+\left(1-\frac{\pi}{2} X\right) C_{c}} \nabla T_{3}\right) d
$$

105 where $d$ is the distance between two crack's lips at the considering point. Suppose that the 106 crack has a spheroidal shape, $d$ is calculated by:

$$
d=2 X \ell \sqrt{1-\left(\frac{\rho}{\ell}\right)^{2}}
$$

107 where $\ell$ and $\rho$ are the radius of the crack and the distance to the crack's center, respectively. 108 The combination of (7) and (8) yields: 


$$
[T]=\left(\frac{C_{s}}{\frac{\pi}{2} X C_{s}+\left(1-\frac{\pi}{2} X\right) C_{c}} \nabla T_{3}\right) 2 X \ell \sqrt{1-\left(\frac{\rho}{\ell}\right)^{2}}
$$

109 It is convenient to introduce also the following dimensionless temperature discontinuity:

$$
[t]=\frac{[T]}{\nabla T_{3}} \frac{1}{\ell}=\left(\frac{2 X C_{s}}{\frac{\pi}{2} X C_{s}+\left(1-\frac{\pi}{2} X\right) C_{c}}\right) \sqrt{1-\left(\frac{\rho}{\ell}\right)^{2}}
$$

110 For the case of conducting crack, i.e. $C_{c}>0$, the limit $X \rightarrow 0$ (penny-shape crack) yields $[t] \rightarrow$ 0 . More precisely, there is no temperature jump across a penny-shape conducting crack. For the case of non-conducting penny-shape crack $\left(C_{c}=0\right)$, equation (10) is simplified (see also

113 Sevostianov, 2006; Vu et al., 2015) as:

$$
[t]=\frac{4}{\pi} \sqrt{1-\left(\frac{\rho}{\ell}\right)^{2}}
$$

114 The maximum value of $[t]=4 / \pi$ is found at the center of the crack $(\rho=0)$. The analytical 115 solution (11) could be considered as a reference to compare with the numerical simulation.

116 Considering the FEM approach for the simulation of this basic problem of heat flow across a 117 single crack, a vertical cylinder containing a horizontal penny-shape crack is analyzed (Fig. 118 2). Unit vertical temperature gradient is applied on the boundary of the cylinder: $T=\underline{\mathrm{e}}_{3} \cdot \underline{\mathrm{z}}$. 119 The dimension of the cylinder is chosen large enough to ensure the far-field boundary condition. The calculation is performed in axis symmetric model thank to the symmetry of the problem. 


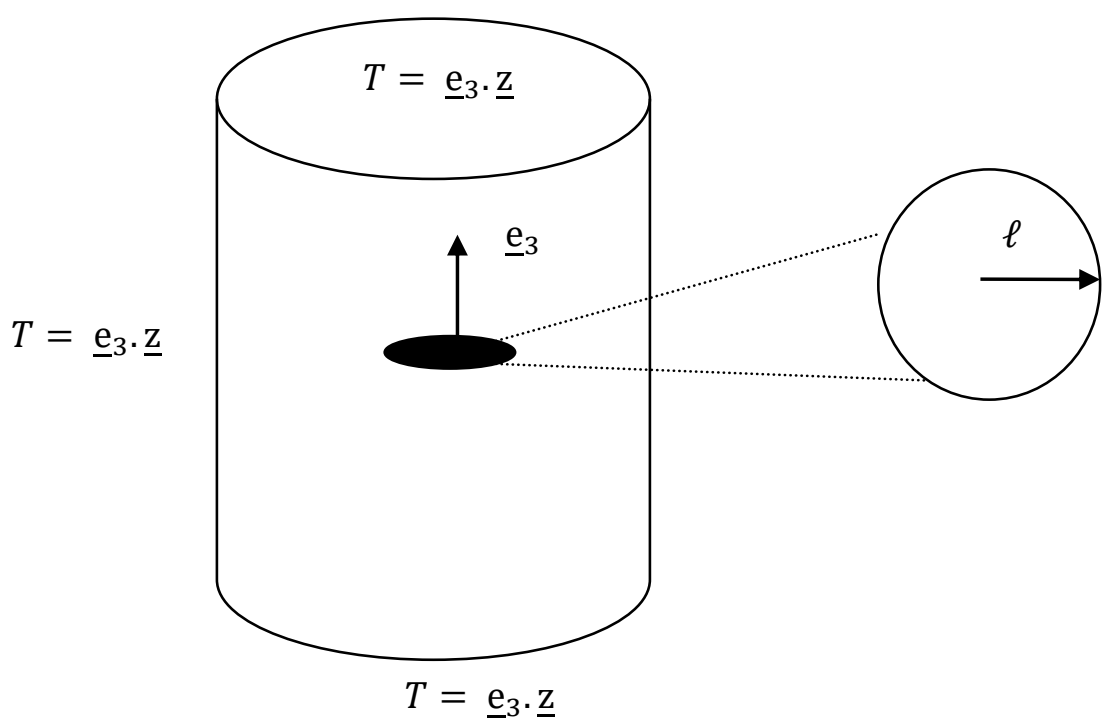

123 Figure 2: Numerical simulation of heat flow across a single crack: geometry and boundary 124 conditions.

125 In this model, the crack is defined by a thin horizontal domain with a given conductivity. Zero 126 conductivity is chosen for the crack's domain when modeling a non-conducting crack. The 127 thickness of the crack domain is chosen small enough to ensure the convergence of the 128 results. It is verified that, a ratio between the thickness and the radius of the crack smaller 129 than 0.005 is enough. The mesh is refined around the crack, therefore a too small crack's 130 thickness will unnecessarily raise the calculation time. The simulation is carried out by using 131 FEM codes Cast3M (Bentejac and Hourdequin, 2005). Fig. 3 displays the mesh (in axis symmetric model), the temperature distribution in the whole domain (left side) and the local vertical temperature gradient across the crack (right side). 
(a)

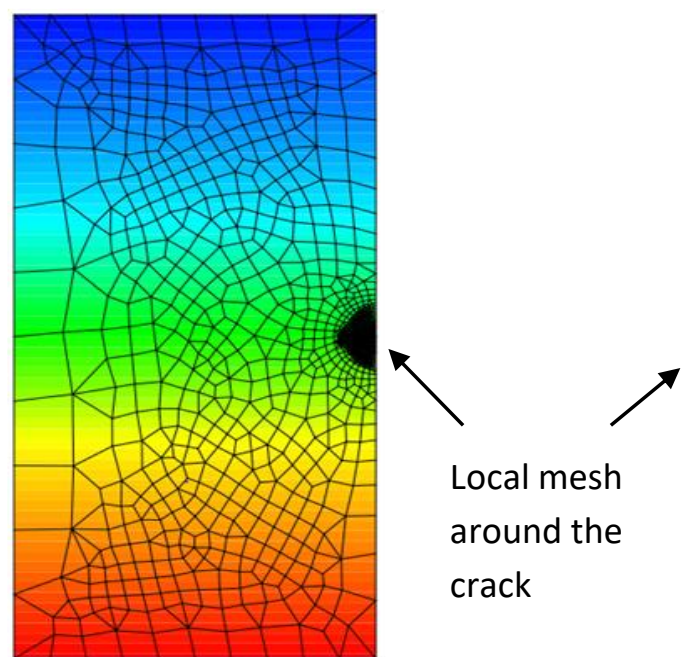

(b)

2. $53 \mathrm{E}+02$

2. $41 \mathrm{E}+02$

$2.29 \mathrm{E}+02$

$2.16 \mathrm{E}+02$

2. $04 \mathrm{E}+02$

1. $92 \mathrm{E}+02$

1. $80 \mathrm{E}+02$

1. $68 \mathrm{E}+02$

1. $56 \mathrm{E}+02$

1. $44 \mathrm{E}+02$

1. $31 \mathrm{E}+02$

1. $19 \mathrm{E}+02$

$1.07 \mathrm{E}+02$

95.

83.

71.

59.

46.

34.

10.

Figure 3: Numerical simulation of heat flow across a single crack: (a) temperature distribution; (b) Local temperature gradient across the crack.

In the particular case of non-conducting crack, the numerical simulation of the temperature jump across the crack is compared with the analytical solution given by the eq. (11). Fig. 4 shows the dimensionless temperature discontinuity along the crack radius. A perfect fit between the numerical and the analytical approaches can be observed.

Note that the solution given by eq. (11) is for non-conducting crack such as open and dry crack. However fluid saturated or partially saturated crack and closed crack are conducting. Fig. 4 shows also the effect of the relative conductivity of the crack and of the surrounding solid matrix on the temperature jump across the crack. For $C_{c} / C_{s} \approx 0.1$, the temperature jump is negligible. For the case of water saturated cracks in rocks (based on data given by Clauser

147 and Huenges, 1995): $C_{c} / C_{s}=C_{\text {water }} / C_{s} \approx 0.1 \div 0.3$. For this case, cracks do not affect the 148 heat flow across the crack in its normal direction.

149 The basic solutions developed for heat flow across a single crack will be employed and generalized in the following to simulate the effective conductivity of a domain containing multiple cracks. 


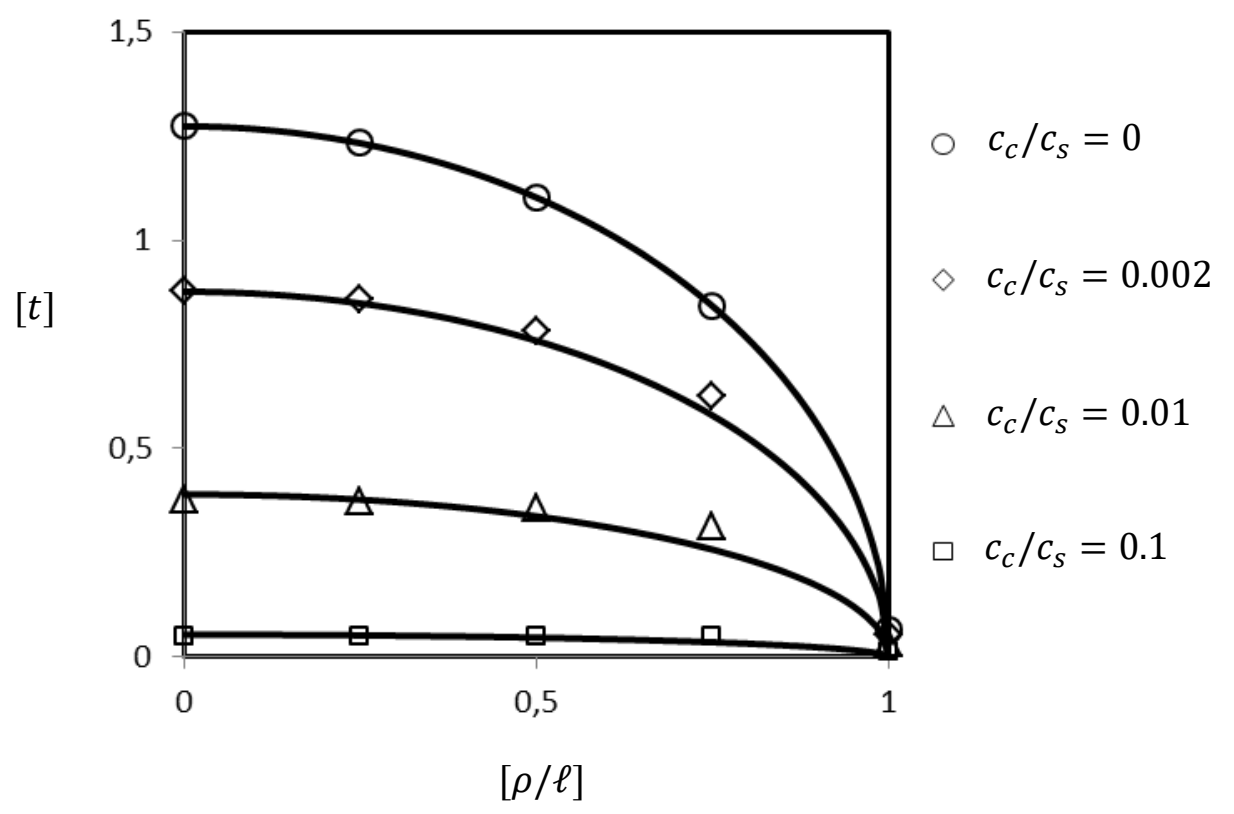

153 Figure 4: Temperature jump across the crack: numerical simulation (the points) and analytical result using eq. (10) (continuous line).

\section{Effective thermal conductivity of cracked media}

This section is dedicated to deriving the effective conductivity of media containing multiple cracks. First, the analytical homogenization-based approaches for the case of nonconducting penny-shape cracks is summarized. Second, a pattern-based numerical approach for both non-conducting and conducting cracks is developed. For non-conducting penny-shape crack, the numerical simulation is compared and constrained with the analytical estimations. The effect of crack conductivity on the effective conductivity of the whole fractured domain is considered at the end of this section.

\subsection{Homogenization-based approaches}

164 The analytical solution (11) of temperature discontinuity across a single crack is a key issue 165 for the estimation of effective thermal conductivity for fractured media. For the case of 166 horizontal parallel cracks in an isotropic homogeneous matrix, the effective conductivity of the medium is transversely isotropic and has on two components: conductivity in the normal 
168 direction to the plan of the cracks $\left(\underline{e}_{3}\right)$ and transversal conductivity. The classical Mori-

169 Tanaka's approach, accounting the fracture interaction, gives (Mori and Tanaka, 1973;

170 Giraud et al., 2007; Nguyen, 2014):

$$
\begin{aligned}
& C_{m t}^{N}=C_{s}+f_{c}\left(C_{c}-C_{s}\right) a^{N}\left(\left(1-f_{c}\right)+f_{c} a^{N}\right)^{-1} \\
& C_{m t}^{T}=C_{s}+f_{c}\left(C_{c}-C_{s}\right) a^{T}\left(\left(1-f_{c}\right)+f_{c} a^{T}\right)^{-1}
\end{aligned}
$$

171 where $C_{m t}^{N}$ and $C_{m t}^{T}$ are the normal and transversal conductivity respectively, $a^{N}$ and $a^{T}$ the

172 two corresponding components of the localization tensor defined by eq. (4)

$$
a^{N}=\frac{C_{s}}{\frac{\pi}{2} X C_{s}+\left(1-\frac{\pi}{2} X\right) C_{c}} ; a^{T}=\frac{C_{s}}{\left(1-\frac{\pi}{4} X\right) C_{s}+\frac{\pi}{4} X C_{c}}
$$

173 The volumetric fraction of the crack is defined by

$$
f_{c}=\frac{4 \pi}{3} N \delta \ell^{2}=\frac{4 \pi}{3} \epsilon X
$$

174 where $N$ is the number of cracks in a unit volume of the medium, $\delta=X \ell$ is haft of the crack's

175 width, $\epsilon=N \ell^{3}$ is the crack density parameter (see also Budiansky and O'connell, 1976).

176

177

178

As discussed in previous section, there is no temperature jump across a penny-shape conducting crack, i.e. the penny-shape conducting cracks do not affect the effective conductivity of the medium. Considering now the case of non-conducting penny-shape cracks $C_{c}=0$ and then substituting (13), (14) into (12) yields:

$$
C_{m t}^{N}=C_{s}\left(1+\frac{8}{3} \epsilon\right)^{-1} ; C_{m t}^{T}=C_{s}
$$

For the case of random orientation distribution of the crack, the conductivity of the whole domain is isotropic:

$$
C_{m t}=\frac{C_{m t}^{N}+2 C_{m t}^{T}}{3}=\frac{C_{s}}{3}\left(2+\left(1+\frac{8}{3} \epsilon\right)^{-1}\right)
$$

The effective conductivity of fractured media can be now estimated for both parallel and random orientation distribution of cracks, by employing (15) and (16). These results account 
184 for the interaction between the cracks but they are limited to the case of non-conducting 185 cracks. More importantly, these solutions do not account for the spatial distribution of the 186 cracks (see Castañeda and Willis, 1995; Bornert et al., 1996).

187 To take into consideration the spatial distribution of the cracks, the results obtained by 188 Gruescu et al. (2007), an extension of the study of Castañeda and Willis (1995), for thermal 189 conductivity $C_{c w}$ of a system of matrix and spheroidal inclusions are considered. A spheroidal 190 distribution of the inclusions was supposed (Fig. 5b).

$$
\begin{aligned}
& C_{c w}^{N}=C_{m}+f_{I} T_{I}^{N}\left(1-f_{I} T_{I}^{N} \frac{1-\frac{\pi}{2} X_{d}}{C_{m}}\right)^{-1} \\
& C_{c w}^{T}=C_{m}+f_{I} T_{I}^{T}\left(1-f_{I} T_{I}^{T} \frac{\frac{\pi}{4} X_{d}}{C_{m}}\right)^{-1}
\end{aligned}
$$

191 with

$$
T_{I}^{N}=\left(\frac{1}{C_{I}-C_{m}}+\frac{1-\frac{\pi}{2} X}{C_{m}}\right)^{-1} ; T_{I}^{T}=\left(\frac{1}{C_{I}-C_{m}}+\frac{\frac{\pi}{4} X}{C_{m}}\right)^{-1}
$$

192 where $C_{m}$ and $C_{I}$ are the conductivity of the matrix and of the inclusions respectively, $f_{I}$ is the 193 volume fraction of the inclusions, $X_{d}$ is the aspect ratio of the distribution which equal to the 194 aspect ratio of the MRP (Fig. 6b) (Castañeda and Willis,1995). A parameter $X_{d}=1$ 195 corresponds to a spherical distribution (Fig. 5a) and a parameter $X_{d} \rightarrow 0$ corresponds to a 196 aligned distribution. 
(a)

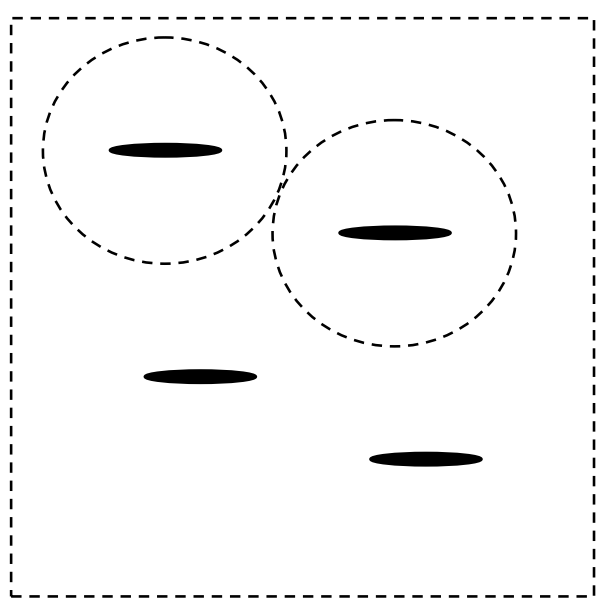

(b)

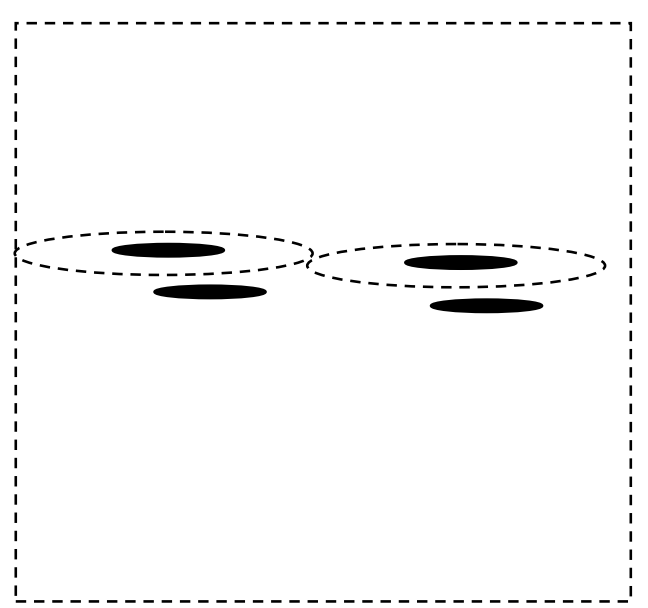

For the case of random orientation distribution of the crack:

$$
C_{c w}=\frac{C_{c w}^{N}+2 C_{c w}^{T}}{3}=C_{s}-\frac{8 C_{s}}{9} \epsilon\left(1+\frac{8}{3} \epsilon\left(1-\frac{\pi}{2} X_{d}\right)\right)^{-1}
$$

202 Note that, for the particular case of aligned distribution of the cracks (the cracks lay closely in 203 the horizontal direction) $X_{d} \rightarrow 0$, (19) and (20) tend to (15) and (16):

$$
\lim _{X_{d} \rightarrow 0} C_{w c}^{N}=C_{S}\left(1-\frac{8}{3} \epsilon\left(1+\frac{8}{3} \epsilon\right)^{-1}\right)=C_{S}\left(1+\frac{8}{3} \epsilon\right)^{-1}
$$

\subsection{Numerical pattern-based method}


PBM is developed to simulate heat flow and effective thermal conductivity of micro-cracked

(a)

$$
\forall \underline{\mathrm{z}} \rightarrow \infty: T=\underline{\nabla T} \cdot \underline{\mathrm{z}}
$$

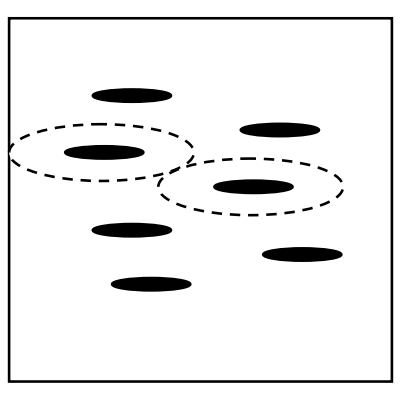

Initial medium (b)
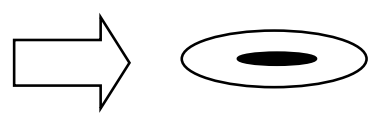

MRP c)

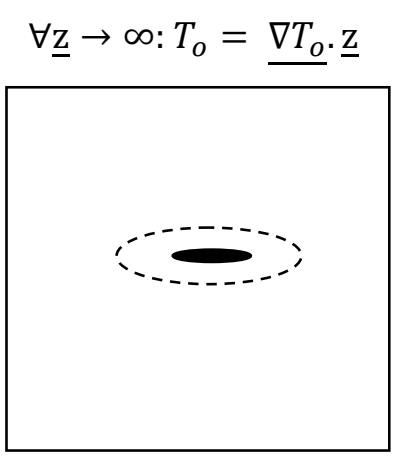

Equivalent medium

Figure 6: MRP (b) of a fractured medium (a) and its equivalent medium for numerical simulation (c). equivalent domain. 


$$
\underline{F}=\frac{1}{V_{M R P}} \int_{M R P} \underline{f} d V
$$

226 Then the effective conductivity is calculated, for the case of parallel cracks, by:

$$
C^{N}=\frac{F_{3}}{\nabla T_{3}} ; C^{T}=C_{S}
$$

227 and for random orientation distribution of cracks by:

$$
C=\frac{C^{N}+2 C^{T}}{3}
$$

228 Fig. 7 shows a comparison between the numerical simulation obtained by current method 229 and the analytical solutions derived in previous section, for the case of parallel non230 conducting cracks. A perfect fit between the numerical approach and the analytical approach 231 (eq. (19)) can be observed. It is to note that both approaches consider the spatial distribution 232 of the cracks. Two distribution was considered: $X_{d}=0.1$ and $X_{d}=0.05$. The numerical results also show that, as presented in eq. (21), when $X_{d}$ tends to zero the conductivity tends to that obtained by the Mori-Tanaka method (eq. (15)).

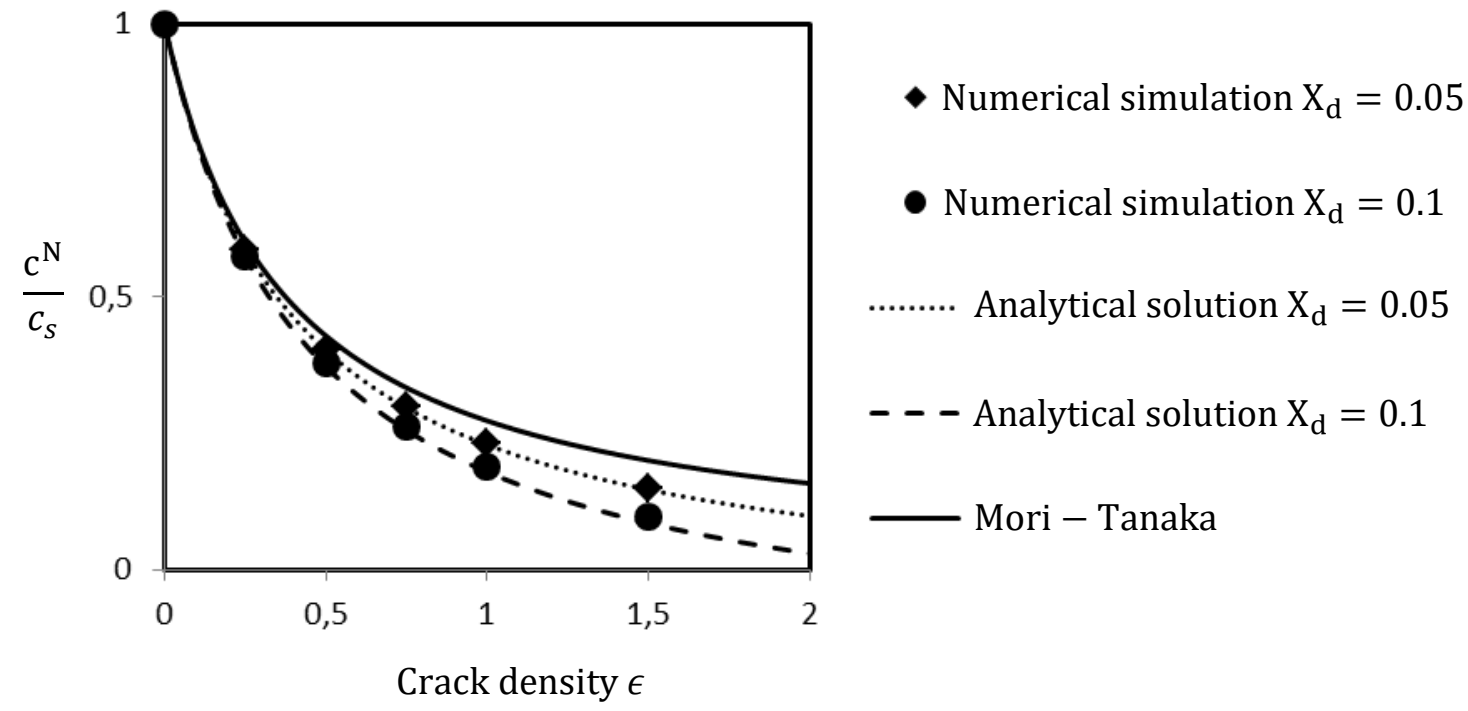

Figure 7: Comparison between the numerical PBM and the analytical homogenization-based approach. 
Note that, differently from the analytical solutions, the numerical method also allows the simulation of the effective conductivity media containing conducting cracks. Fig. 8 shows the effect of the crack conductivity on the overall conductivity of the medium. The simulation suggests that, for $C_{c} / C_{s} \geq 0.1$, the effect of cracks on the overall conductivity of the fractured media is weak.

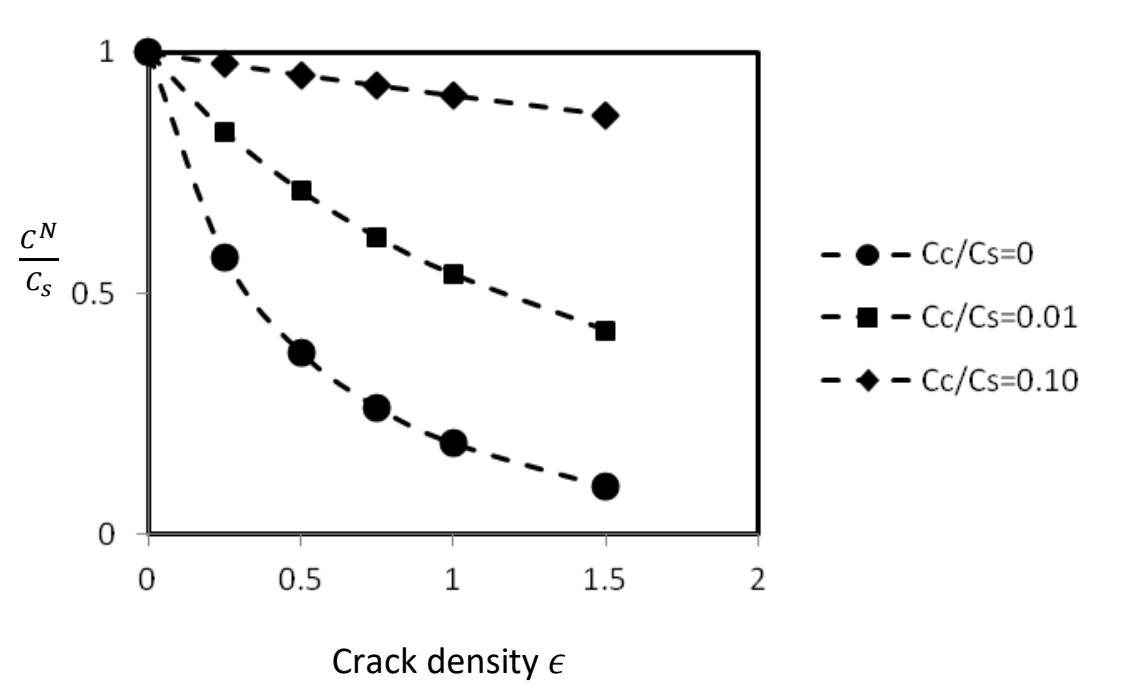

Figure 8: Effect of crack conductivity and crack density on overall conductivity of fractured media: numerical simulations.

\section{Conclusions}

Firstly, heat flow across a single crack is analyzed by both analytical and numerical methods.

A closed-form solution is derived for the temperature jump across a single non-conducting crack under homogeneous gradient far-field boundary condition. This analytical formulation is then validated by the FEM simulation. The effect of crack conductivity on the temperature discontinuity is also analyzed by the numerical method. It is shown that for crack of high conductivity (for example water saturated crack), the temperature jump across the crack can be negligible and the crack affects weakly the heat flow through the whole medium.

Secondly, the basic result of heat flow across a single crack is extended for the case of 255 multiple cracks. Homogenization-based analytical approaches and PBM are employed to 
simulate effective thermal conductivity of fractured materials. Both isotropic and transversely isotropic (parallel cracks) cases are considered. The spatial distribution of the cracks is also taken into account in the analytical and numerical methods. In the particular case of nonconducting crack, the numerical and analytical methods fit perfectly together. When the distribution of the crack is aligned, both approaches tend to the analytical solution developed based on the Mori-Tanaka scheme.

The numerical PBM allows also the simulation of the effect of the crack conductivity on the overall conductivity of the fractured media. It is demonstrated that, for cracks with conductivity equal to of about $10 \%$ of the conductivity of the surrounding solid matrix (for example water saturated rocks), the effect of the cracks system on the overall conductivity of the fractured media can be negligible.

\section{References}

Tang, A. M., \& Cui, Y. J. (2009). Modelling the thermo-mechanical volume change behaviour of compacted expansive clays. arXiv preprint arXiv:0904.3614.

Tang, A. M., Cui, Y. J., \& Le, T. T. (2008). A study on the thermal conductivity of compacted bentonites. Applied Clay Science, 41(3), 181-189.

Bentejac, F., \& Hourdequin, N. (2005). TOUTATIS: An Application of the Cast3m Finite Element Code for PCI Three-Dimensional Modelling. In Pellet-Clad Interaction in Water Reactor Fuels.

Bornert, M. (1996). A generalized pattern-based self-consistent scheme. Computational Materials Science, 5(1), 17-31.

Bornert, M., Stolz, C., \& Zaoui, A. (1996). Morphologically representative pattern-based bounding in elasticity. Journal of the Mechanics and Physics of Solids, 44(3), 307-331.

Budiansky, B., \& O'connell, R. J. (1976). Elastic moduli of a cracked solid. International Journal of Solids and Structures, 12(2), 81-97. 
281 Camacho, G. T., \& Ortiz, M. (1996). Computational modelling of impact damage in brittle 282 materials. International Journal of solids and structures, 33(20), 2899-2938.

283 Castañeda, P. P., \& Willis, J. R. (1995). The effect of spatial distribution on the effective

284

285

286

287

288

289

290

291

292

293

294

295

296

297

298

299

300

301

302

303

304

305

306

307

behavior of composite materials and cracked media. Journal of the Mechanics and Physics of Solids, 43(12), 1919-1951.

Clauser, C., \& Huenges, E. (1995). Thermal conductivity of rocks and minerals. Rock physics \& phase relations: A handbook of physical constants, 105-126.

Cui, Y. J., Tang, A. M., Qian, L. X., Ye, W. M., \& Chen, B. (2011). Thermal-mechanical behavior of compacted GMZ Bentonite. Soils and foundations,51(6), 1065-1074.

Dormieux, L., Kondo, D., \& Ulm, F. J. (2006). Microporomechanics. John Wiley \& Sons.

Eshelby, J. (1957). The determination of the elastic field of an ellipsoidal inclusion and related problems. Proc. R. Soc. London, A 241, 376-396.

Giraud, A., Gruescu, C., Do, D. P., Homand, F., \& Kondo, D. (2007). Effective thermal conductivity of transversely isotropic media with arbitrary oriented ellipsoidal inhomogeneities. International Journal of Solids and Structures, 44(9), 2627-2647.

Gruescu, C., Giraud, A., Homand, F., Kondo, D., \& Do, D. P. (2007). Effective thermal conductivity of partially saturated porous rocks. International Journal of Solids and Structures, 44(3), 811-833.

Mori, T., \& Tanaka, K. (1973). Average stress in matrix and average elastic energy of materials with misfitting inclusions. Acta metallurgica, 21(5), 571-574.

Nguyen, ST (2014a). Micromechanical approach for electrical resistivity and conductivity of sandstone. Journal of Applied Geophysics, 111, 135-140.

Nguyen, ST (2014b) Generalized Kelvin model for micro-cracked viscoelastic materials. Engineering Fracture Mechanics, 127, 226-234.

Nguyen, S. T., \& Dormieux, L. (2014). Propagation of micro-cracks in viscoelastic materials: Analytical and numerical methods. International Journal of Damage Mechanics, 1056789514539715. 
308 Nguyen, ST, Dormieux, L, Le Pape, Y \& Sanahuja, J (2011) A Burger model for the effective 309 behavior of a microcracked viscoelastic solid. International Journal of Damage Mechanics, $31020(8), 1116-1129$.

311 Nguyen, ST, Vu, MH, \& Vu, MN (2015a). Extended analytical approach for electrical anisotropy of geomaterials. Journal of Applied Geophysics, 123, 211-217. Nguyen, S. T., Vu, M. H., \& Vu, M. N. (2015b). Equivalent porous medium for modeling of the elastic and the sonic properties of sandstones. Journal of Applied Geophysics, 120, 1-6. Nguyen, TN, Nguyen, ST, Vu, MH, \& Vu, MN (2015c). Effective viscoelastic properties of micro-cracked heterogeneous materials. International Journal of Damage Mechanics, 1056789515605557.

Pouya, A., Vu, M. N., Ghabezloo, S., \& Bendjeddou, Z. (2013). Effective permeability of cracked unsaturated porous materials. International Journal of Solids and Structures, 50(20), 3297-3307.

321 Stolz, C., \& Zaoui, A. (1991). Analyse morphologique et approches variationnelles du 322 comportement d'un milieu élastique hétérogène. Comptes rendus de l'Académie des sciences. Série 2, Mécanique, Physique, Chimie, Sciences de l'univers, Sciences de la Terre, 312(3), 143-150. Vu, M. N., Nguyen, S. T., Vu, M. H., Tang, A. M., To, V. T. (2015). Heat conduction and thermal conductivity of 3D cracked media. International Journal of Heat and Mass Transfer $32789,1119-1126$.

328 Zimmerman, R. W. (1989). Thermal conductivity of fluid-saturated rocks. Journal of 329 Petroleum Science and Engineering, 3(3), 219-227. 